\title{
Axonal Neurofilaments Control Multiple Fiber Properties But Do Not Influence Structure or Spacing of Nodes of Ranvier
}

\author{
Rodolphe Perrot, ${ }^{1}$ Pierre Lonchampt, ${ }^{1}$ Alan C. Peterson, ${ }^{2}$ and Joël Eyer ${ }^{1}$ \\ ${ }^{1}$ Laboratoire de Neurobiologie et Transgenese, Unité Propre de Recherche de l'Enseignement Supérieur-Equipe d'Accueil 3143, Institut National de la Santé \\ et de la Recherche Médicale, Bâtiment Monteclair, Centre Hospitalier Universitaire, Angers, 49033, France, and ²Molecular Oncology Group, McGill \\ University, Royal Victoria Hospital, Montreal, Quebec, Canada H3A1A1
}

In the vertebrate nervous system, axon calibers correlate positively with myelin sheath dimensions and electrophysiological parameters including action potential amplitude and conduction velocity. Neurofilaments, a prominent component of the neuronal cytoskeleton, are required by axons to support their normal radial growth. To distinguish between fiber features that arise in response to absolute axon caliber and those that are under autonomous control, we investigated transgenic mice in which neurofilaments are sequestered in neuronal cell bodies. The neurofilament deficient axons in such mice achieve mature calibers only $50 \%$ of normal and have altered conduction properties. We show here that this primary axonal defect also induces multiple changes in myelin sheath composition and radial dimensions. Remarkably, other fundamental fiber features, including internodal spacing and the architecture and composition of nodes of Ranvier, remain unaltered. Thus, many fiber characteristics are controlled through mechanisms operating independently of absolute axon caliber and the neurofilament cytoskeleton.

Key words: neurofilaments; node of Ranvier; myelin; conduction velocity; internodal length; transgenic mice

\section{Introduction}

Along myelinated fibers, excitable membrane is limited to nodes of Ranvier, a restriction that imposes rapid and energy efficient saltatory conduction of action potentials (Stampfli, 1954). Conduction properties are influenced further by axon caliber (Sakaguchi et al., 1993; Kriz et al. 2000), internodal length (Court et al., 2004), and the molecular structure of nodes of Ranvier (Bhat et al., 2001; Boyle et al., 2001). Early ultrastructural investigations demonstrated that myelinated fibers develop stereotyped ratios between axon caliber, myelin thickness, and internodal distances. With increasing axon calibers, myelin sheaths are proportionally thicker and longer. Notably, internodal lengths are $\sim 100$-fold the caliber of the ensheathed axon (Rushton, 1951; Fried et al., 1982). Although such conserved relationships confer fundamental

\footnotetext{
Received March 19, 2007; revised June 30, 2007; accepted July 12, 2007.

This work was supported by grants from the Association Française contre les Myopathies and Association pour la Recherche sur la Sclerose en Plaques (J.E.), and the MS Society of Canada (A.P.). R.P. was supported by Angers Agglomeration. We thank Prof. E. Peles (Weizmann Institute of Science, Rehovot, Israel), J. Salzer (New York University, New York, NY), and H. Freidman (McGill University, Montreal, Quebec, Canada) for critical reading of this manuscript and helpful scientific suggestions. We gratefully acknowledge P. Valera, I. Tretjakoff, and M. Attiwell for their excellent technical assistance. We are grateful to the Service Commun d'Imageries et d'Analyses Microscopiques de l'Universite d'Angers for assistance in electron and confocal microscopy, and to P. Chiron (animal facility)

Correspondence should be addressed to Joël Eyer, Laboratoire Neurobiologie and Transgenese, UPRES EA3143, Institut National de la Santé et de la Recherche Médicale, CHU, Angers 49033, France. E-mail: joel.eyer@univ-angers.fr.

DOI:10.1523/JNEUROSCI.1224-07.2007

Copyright $\odot 2007$ Society for Neuroscience $\quad$ 0270-6474/07/279573-12\$15.00/0
}

properties to the nervous system, the molecular mechanisms engaged to establish and maintain them are not well understood.

One model accommodating the multiple size and spacing relationships between axons and myelin sheaths suggests that myelin-forming cells control the distribution of nodes whereas the axonal cytoskeleton serves to control axon calibers and anchor nodal components (Poliak and Peles, 2003; Sherman and Brophy, 2005). Notably, sodium channels are linked to ankyrin $G$ (Lemaillet et al., 2003), which connects to the actin axoskeleton via $\beta I V$-spectrin. Caspr/paranodin and contactin are concentrated in the paranodal axolemma and anchored to the actin axoskeleton via protein 4.1B (Denisenko-Nehrbass et al., 2003). The Shaker-type potassium channels are concentrated at the juxtaparanode adjacent to compact myelin. Finally, neurofilaments (NFs) are prominent cytoskeletal components in the internodal domain of all large caliber axons. They assemble from three subunits and bear long sidearms that are highly phosphorylated in myelinated domains (de Waegh et al., 1992; Mata et al., 1992; Garcia et al., 2003; Rao et al., 2003). As demonstrated in multiple animal models with absent or disorganized NFs, they play an essential role in axon radial growth (Cleveland et al. 1991; Sakaguchi et al., 1993; Elder et al., 1999; Jacomy et al., 1999). However, because mice null for heavy NF subunit (NFH) achieve normal axonal calibers but conduct action potentials at reduced speeds, NFs control more than the physical dimensions of axons (Kriz et al., 2000).

In this investigation, we use a mouse model in which axons mature in the absence of an NF cytoskeleton (Eyer and Peterson, 
1994). As expected, radial growth of axons throughout the CNS and PNS is attenuated and conduction is slowed. Different perturbations in myelin sheath dimensions and composition are observed in the CNS and PNS. Despite such axonal and myelin changes, the organization and composition of nodes of Ranvier appear unaltered. These results indicate that fiber phenotypes are controlled by multiple mechanisms. One operates independently of NFs, axon caliber, and myelin thickness to position and assembles nodes of Ranvier. The other depends on NFs and operates in the radial dimensions increasing axon calibers to achieve adequate conduction velocities.

\section{Materials and Methods}

Mice. The production of the NFH- $\beta$-galactosidase (NFL-LacZ) transgenic mice was described previously (Eyer and Peterson, 1994). Subsequently, the transgene was maintained as a randomly bred line derived from $\mathrm{B} 6 \mathrm{C} 3 \mathrm{~F} 1 \mathrm{~s}$ and by backcrossing to multiple inbred strains. In this investigation, we used the $\mathrm{B} 6 \mathrm{C} 3 \mathrm{~F} 1$ derivatives and, for the serial section study, backcross C57BL/6 mice.

Optic and electron microscopy. Samples were prepared for embedding in Epon as described previously (Eyer and Peterson, 1994). For light microscopy, $1-\mu \mathrm{m}$-thick sections were stained with methylene blue-azur II. For electron microscopy, ultrathin $(75 \mathrm{~nm})$ sections were collected on copper grids, counterstained with 5\% uranyl acetate and lead citrate, and examined using a JEOL (Peabody, MA) JEM 2010 electron microscope.

Preparation of teased sciatic fibers. Sciatic nerves from 3-month-old mice were isolated and rinsed in phosphate buffer. They were teased onto Star Frost slides (Knittel Gläser, Braunschweig, Germany) and allowed to dry. Fibers were incubated with $1 \%$ osmium tetroxide for $1 \mathrm{~h}$ at $4^{\circ} \mathrm{C}$, washed in water, and counterstained with $0.1 \mathrm{M} \mathrm{FeCl}_{3} / 2 \mathrm{M}$ acetic acid for $1 \mathrm{~h}$ and $0.02 \mathrm{M} \mathrm{K}_{3} \mathrm{Fe}, \mathrm{pH} 4$, for $30 \mathrm{~min}$. Slides were mounted with Aquatex (Merck, Whitehouse Station, NJ) and analyzed using a Leica (Oberkochen, Germany) DMR microscope and IM500 software.

Isolation of myelin. Tissues were removed from 4-month-old mice, weighed, homogenized in $0.25 \mathrm{M}$ sucrose, and centrifuged for $10 \mathrm{~min}$ at $500 \times g$ at $4^{\circ} \mathrm{C}$. The supernatant was then centrifuged for $10 \mathrm{~min}$ at $11000 \times g$ at $4^{\circ} \mathrm{C}$, and the resulting pellet was resuspended in 10 volumes of $0.25 \mathrm{~m}$ sucrose. The same volume of $0.88 \mathrm{M}$ sucrose was added delicately below, and centrifuged for $3 \mathrm{~h}$ at $100000 \times g$ at $4^{\circ} \mathrm{C}$. Myelin was collected at the interface between the two sucrose layers, and resedimented at $15000 \times g$ for $30 \mathrm{~min}$ at $4^{\circ} \mathrm{C}$. The pellet was homogenized in 10 volumes of ice-cold water and centrifuged at $15000 \times g$ for $30 \mathrm{~min}$ at $4^{\circ} \mathrm{C}$. This step was repeated twice. The final pellet was weighed.

Western blot and immunofluorescence analysis. Tissues collected from 4-month-old animals were homogenized in $320 \mathrm{~mm}$ sucrose and protease inhibitors, and centrifuged for $5 \mathrm{~min}$ at $2000 \times g$ at room temperature. This crude extract was then centrifuged at $100,000 \times g$ for $1 \mathrm{~h}$ and the resulting pellet was resuspended in the same solution. The amount of proteins was determined using the BCA Protein assay kit (Pierce, Rockford, IL). Proteins $(50 \mu \mathrm{g})$ were solubilized in Laemmli buffer (Laemmli, 1970), heat-denatured, electrophoresed on 7.5\% SDS-polyacrylamide gels, and electrotransferred onto nitrocellulose membranes (Millipore, Lake Placid, NY) for immunoblotting. Membranes were blocked overnight using $10 \%$ dry milk diluted in TBS and then incubated with primary antibodies.

Immunofluorescence staining of frozen tissue samples was performed as described by Arroyo et al. (2002) and analyzed with a confocal microscope (BX50 with Fluoview software 3.0; Olympus, Tokyo, Japan). Concentrations of antibodies used for Western blot (WB) and immunofluorescence (IF) were as follows: mouse anti- $\beta$ III-tubulin (1:1000; Sigma, St. Louis, MO); mouse anti-pan-sodium channel (WB, 1:1000; IF, 1:100; Sigma); rabbit anti-voltage-gated sodium channel 1.2 (Nav1.2; 1:100; Alomone Labs, Jerusalem, Israel); rabbit anti-Nav1.6 (1:100; Alomone Labs); rabbit anti- $\beta$ IV-spectrin (WB, 1:500; IF, 1:50; gift from M. Solimena, University of Technology Dresden, Dresden, Germany); chicken anti- $\beta$ IV-spectrin (1:400; gift from M. Komada, Tokyo Institute of Technology, Yokohama, Japan); mouse anti-neuregulin 1 type I (1:200; Thermo Fisher Scientific, Fremont, CA); rabbit anti-neuregulin 1 type III
(1:200; gift from C. Lay, Scripps Research Institute, La Jolla, CA); rabbit anti-erbB2 (1:200; Santa Cruz Biotechnology, Santa Cruz, CA); mouse anti-Shaker-type voltage-gated potassium channel (Kv1.1; 1:100; Millipore); rabbit anti-Caspr (1:2000; gift from J.-A. Girault, Institut National de la Santé et de la Recherche Médicale U536, Institut du Fer à Moulin, Paris, France).

For Western blots, peroxydase-conjugated secondary antibodies (1: 1000; Dako, High Wycombe, UK) were revealed using the enhanced chemiluminescence (ECL) detection system (GE Healthcare BioSciences, Arlington Heights, IL) and radiographic films (Hyperfilm ECL; GE Healthcare Bio-Sciences). Band intensities were determined using $\mathrm{NIH}$-Image software and quantifications were normalized against levels of tubulin. Control to transgenic ratios were calculated from at least three experiments. For immunofluorescence, secondary antibodies (diluted 1:200) were Alexa Fluor 488 goat anti-mouse IgG antibodies, Alexa Fluor 568 goat anti-rabbit IgG antibodies, and Alexa Fluor 488 goat antichicken IgG antibodies (Invitrogen, Eugene, OR).

Morphometrical analysis. Axonal caliber and myelin thickness were measured on methylen blue-azur-II-stained semithin sections of sciatic nerve and spinal cord. The cross-sectional axonal area of myelinated axons was measured and axonal caliber was determined by the diameter of a circle of area equivalent to each axon. The g ratio was determined by dividing the diameter of the axon by the diameter of the fiber (axon with myelin). Nodal and paranodal length were measured on longitudinal sections of the spinal cord or the sciatic nerve following, respectively, anti-pan sodium channel and anti-Caspr immunolabeling. Measurements were done using a Leica DMR microscope $(100 \times$ objective) and the Leica QWin software or the Leica IM500 software. The internodal length was measured as described in the results section. Significant differences were determined using simple or paired $t$ test.

\section{Results}

\section{CNS and PNS fibers regulate myelin thickness differently in response to reduced axon calibers}

The NFH- $\beta$-galactosidase fusion gene is controlled by NFH regulatory sequences. Consequently, it initiates expression in prenatal transgenic mice and expression is maintained throughout life. Axons in such mice are constitutively deficient for NFs, undergo limited radial growth, and maintain inappropriately small calibers. As reported previously (Eyer and Peterson, 1994), mature motor axons in these mice have calibers approximating $50 \%$ of normal; average axon calibers in the lumbar 4 (L4) ventral roots from 110-d-old control and transgenic littermates were, respectively, $4.56 \mu \mathrm{m}$ (1027 fibers) and $2.61 \mu \mathrm{m}$ (927 fibers). Here, we extend this quantitative analysis to include more distal PNS axon segments and CNS fibers. Cross sections from sciatic nerves of 4-month-old mice were obtained $2-3 \mathrm{~mm}$ distal from the sciatic notch. Average axon calibers in transgenic sciatic nerves were only $53 \%$ of normal with respective values of $1.98 \mu \mathrm{m}(2228$ fibers) and $3.73 \mu \mathrm{m}$ (2002 fibers) (Fig. 1A,B).

To evaluate the extent of axon caliber reduction in CNS fibers, cross sections were obtained from the thoracic spinal cord of 5-month-old mice. On each side of the midline, starting at the deep medial boundary of the ventral funiculus and extending ventrally and laterally, a contiguous population of fibers was measured. In this unbiased sampling approach, fibers of all calibers were included. The mean axon diameter in control samples was $3.05 \mu \mathrm{m}$ (2095 fibers) and $1.65 \mu \mathrm{m}$ (2073 fibers) in transgenic samples (Fig. $1 A, B$ ). Similar to the PNS, the average caliber of NF-deficient CNS axons was only $54 \%$ of normal. However, the caliber of the smallest myelinated axons was similar in transgenic and control samples.

Axons and their associated myelinating cells develop and maintain a similar axon caliber-sheath thickness ratio regardless of absolute axon calibers or their trajectory in the CNS or PNS compartment. Although a similar reduction in axon calibers is 


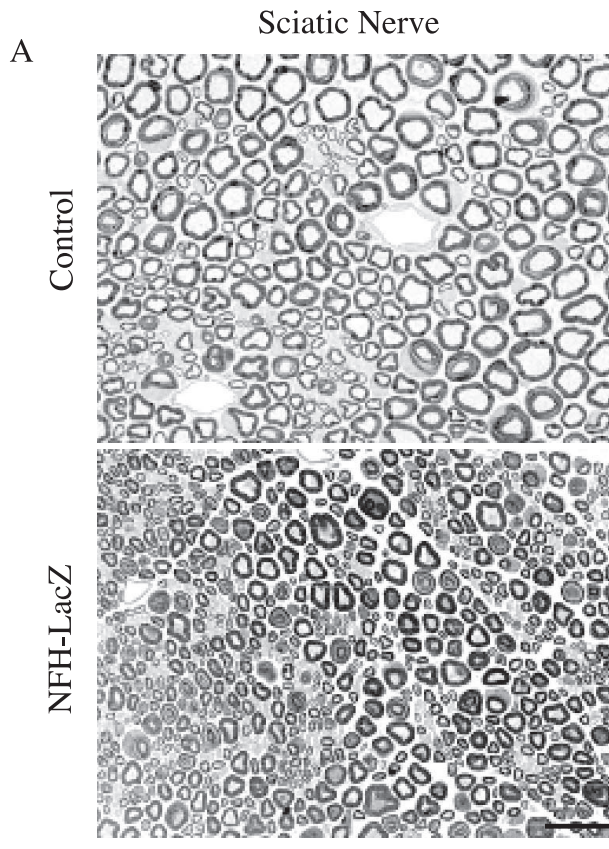

B

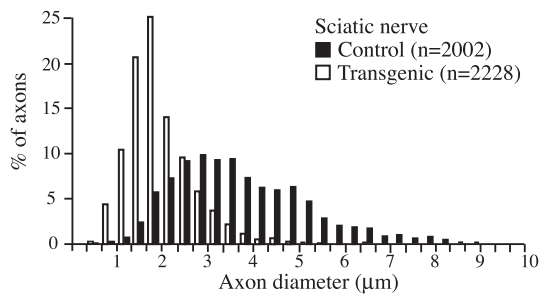

C
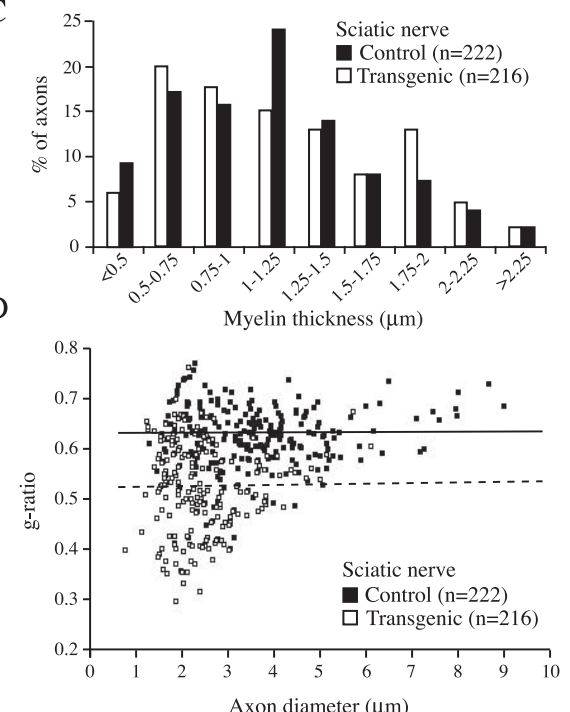

Spinal Cord
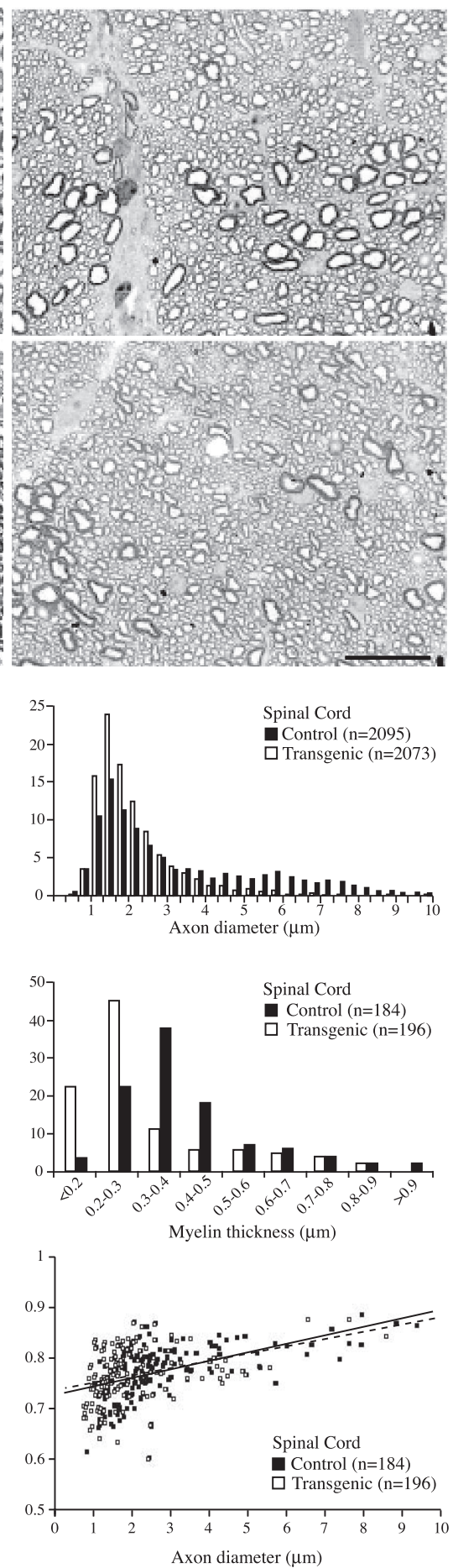

Figure 1. The size of myelinated fibers is reduced in NFH-LaCZ mice compared with control animals in both the CNS and PNS. $A$, Typical cross section views of sciatic nerves ( 4 months of age) and spinal cords (5 months of age) from wild-type and NFH-LacZ mice. Scale bars, $20 \mu \mathrm{m}$. $\boldsymbol{B}$, Measurement of the axonal calibers of sciatic nerves and spinal cords indicates that the size of axons is dramatically reduced in the absence of NFs (control samples in black, and transgenic in white bars). Note the shift to smaller diameter fibers in transgenic mice. C, Myelinating cells respond differently to the axonal NF deficiency in the CNS and PNS of NFH-LacZ mice. The myelin thickness is not significantly modified in sciatic nerve from 4-month-old transgenic mice (white bars) compared with controls (black bars), but is proportionately reduced to the axonal caliber in NFH-LacZ spinal cord. D, Consequently, gratios were reduced in transgenicsciaticnerve (whitesquares) compared with controls (blacksquares), but unaffected between transgenic (white squares) and control (black squares) spinal cords.

observed in the CNS and PNS of NFH-LacZ mice, myelination levels in the two sites differ markedly (Fig. 1C,D). The caliberreduced axons in the CNS are invested with proportionally thinner myelin such that the average g ratio (axon diameter/fiber diameter) is similar to control values $(0.76 \pm 0.05$ vs $0.78 \pm 0.05)$. In contrast, and as observed for spinal root fibers (Eyer and Peterson, 1994), axons in the sciatic nerves of NFH-LacZ transgenic mice recruit inappropriately thick myelin relative to their absolute calibers (Fig. $1 A, C, D$ ), resulting in an average g ratio of $0.52 \pm$ 0.09 vs $0.63 \pm 0.06$ in control $(p<0.001)$. As revealed in Figure $1 D$, this relative hypermyelination occurs for axons of all calibers (Fig. 1D). Despite the affected myelination, additional malformations were not obvious.

The difference in relative myelin thickness observed between the CNS and PNS fibers of transgenic mice is reflected in the total amount of myelin isolated from the two tissues by sucrose gradient techniques. The amount of myelin extracted from control and NFH-LacZ sciatic-nerve samples was similar (605 and $588 \mathrm{mg}$ of myelin/g of tissue, respectively). In contrast, the amount of myelin extracted from control brains (158 mg of myelin/g) was more than twice that recovered from transgenic brains (62 mg of myelin/g). A similar difference was observed for transgenic and control spinal cord samples (471 and 192 $\mathrm{mg}$ of myelin/g, respectively). These observations require that oligodendrocytes and Schwann cells respond differently to the lack of axonal NFs and/or reduced axon calibers.

\section{Composition and structure of myelin} sheaths in CNS and PNS fibers of NFHLacZ transgenic mice

To investigate whether the differences in CNS and PNS myelin extended to major myelin components, we compared the relative accumulation of myelin proteins by Western blot analysis. Equal amounts of crude myelin extract isolated from brains and spinal cords were loaded per well. Equivalent amounts of proteolipid protein (PLP), myelin basic protein (MBP), CNPase, and CD9 were detected in transgenic and control samples. Similarly, the quantity of MBP, CNPase, and CD9 appeared to be similar in transgenic and control sciatic-nerve samples. In contrast, $\mathrm{P} 0$ accumulation was reduced by $23 \pm 8 \%$ in transgenic sciatic-nerve myelin extracts (Fig. 2A, Table 1).

The periodicity of myelin is highly conserved among vertebrates (Hildebrand, 1972). Because experimental alterations in P0 expression are known to alter the periodicity of both peripheral and central myelin (Giese et al., 1992; Yin et al. 2006), we compared the periodicity of myelin sheaths of similar thickness in the sciatic nerves of 3-month-old transgenic and control mice. Consistent with previous studies, the interpe- 
A

\begin{tabular}{|c|c|c|c|c|c|c|}
\hline & \multicolumn{2}{|c|}{ Brain } & \multicolumn{2}{|c|}{ Spinal Cord } & & Sciatic Nerve \\
\hline & - & + & & + & & + \\
\hline PLP & $\infty$ & $\infty$ & $\infty$ & & $\mathrm{P}_{0}$ & \\
\hline MBP & & $-\infty$ & & & MBP & 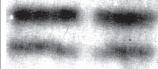 \\
\hline CNPase & - & $=$ & - & - & CNPase & -1 \\
\hline CD9 & $=$ & 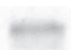 & $=$ & $m$ & CD9 & $-\infty$ \\
\hline Tub & $\infty$ & & & & Tub & 0 \\
\hline
\end{tabular}

B
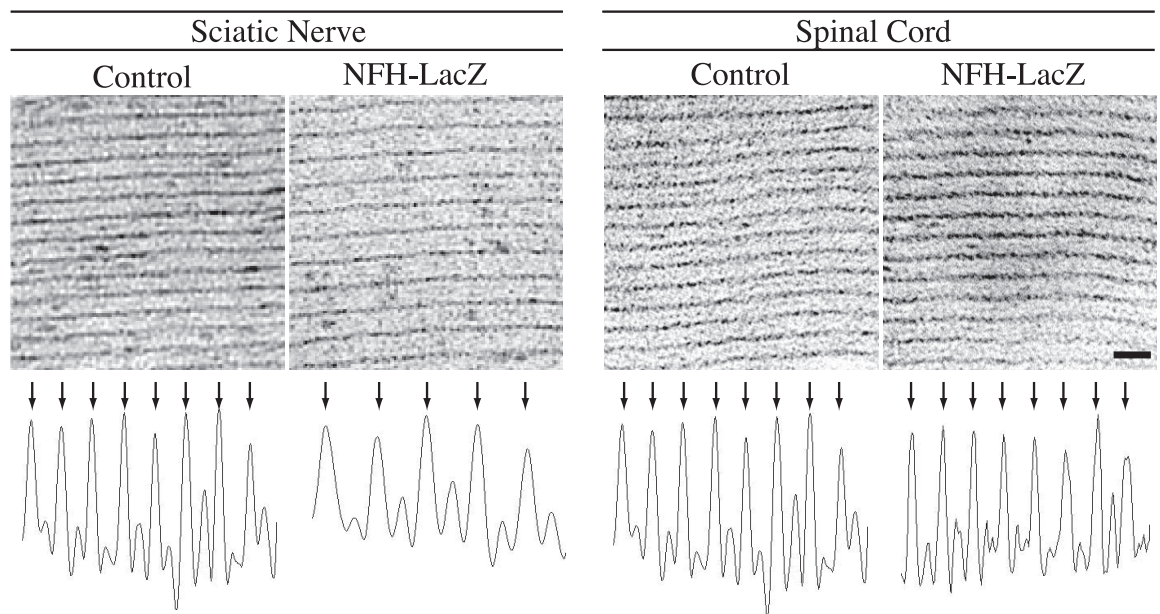

$\mathrm{C}$

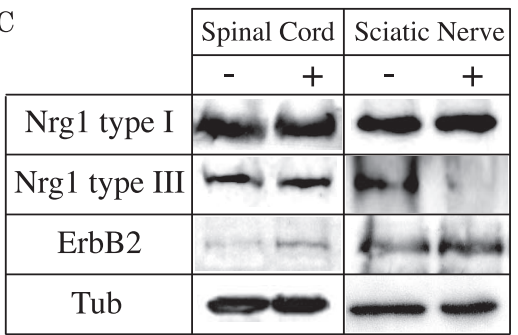

Figure 2. Composition and ultrastructure of myelin in NFH-LacZ mice. A, Western blot analysis of myelin associated proteins. The crude myelin extracts from brain, spinal cord, and sciatic nerve of 3-month-old control $(-)$ and NFH-LacZ $(+)$ mice were analyzed by Western blot using antibodies recognizing P0, PLP, MBP, CNPase, CD9, and tubulin (Tub) for control. The two bands shown for MBP are the 14 and $17 \mathrm{kDa}$ isoforms and were combined for quantification. The amount of these proteins was unaffected in transgenic mice, except for $\mathrm{PO}$ that was decreased by $23 \pm 8 \%$ in NFH-LacZ sciatic nerves. $\boldsymbol{B}$, Electron micrographs showing periodicity of myelin in the sciatic nerve and the spinal cord of 3-month-old mice. Scale bar, $20 \mathrm{~nm}$. Densitometry graphs described the myelin period in sciatic nerve and spinal cord from control and transgenic mice. Arrows indicate deflections corresponding to major dense lines. Note the difference of lamellar spacing between control and NFH-LacZ sciatic nerves. C, Western blot analysis of Nrg1 types I and III, and erbB2 in total protein lysates from control and NFH-LacZ sciatic nerve and spinal cord. Expression of Nrg1 type III is reduced in NFH-LacZ sciatic nerve whereas erbB2 is increased in NFH-LacZ spinal cord.

was also suggested (Michailov et al., 2004). To determine whether the altered axon caliber-myelin relationships in NFH-LacZ mice could be achieved through this pathway, we compared the amount of $\mathrm{Nrg} 1$ type I and type III and their coreceptor erbB2 accumulated in sciatic nerves and spinal cords of 3-month-old transgenic and control mice (Fig. 2C). The amounts of Nrgl type I in CNS and PNS samples were similar between the two genotypes and no differences were observed in the amounts of Nrg1 type III accumulated in transgenic and control spinal cord samples. However, the amount of Nrgl type III accumulated in transgenic sciatic nerves was significantly reduced by $50 \pm 18 \%$ $(p<0.05)$. Finally, although no change was found for erbB2 expression in transgenic sciatic nerves, levels of erbB2 were increased by $32 \pm 8 \%$ in transgenic spinal cords $(p<0.05)$ (Fig. 2C). Although these observations highlight the intriguing possibility that signaling based on $\mathrm{Nrg} 1$ and erbB2 may be affected in NF null fibers, they also demonstrate that this mechanism is not shared, at least in its entirety, by CNS and PNS fibers.

\section{Internodal lengths in both PNS and CNS fibers remain normal despite reduced axonal calibers and altered myelin thickness}

Both internodal distance and myelin thickness are proportional to axon caliber (Rushton, 1951; Fried et al., 1982). If the absolute size of axons plays an important role in this relationship, the caliberreduced axons in NFH-LacZ mice should maintain correspondingly shorter internodes. However, if internodal distance was unaffected, it would suggest that the mechanism determining nodal spacing operates independently of absolute axonal caliber, myelin thickness, and axonal NFs. Multiple strategies were used to estimate and measure internodal lengths in both the CNS and PNS of transgenic and control mice.

Because Schwann cells elaborate only one myelin sheath, any deviation from the normal internodal length would cause a

riodic distance observed in the $\mathrm{P} 0$-deficient myelin of the $\mathrm{NFH}$ LacZ mice $(13.8 \pm 1.1 \mathrm{~nm})$ was increased relative to control values $(12.2 \pm 0.9 \mathrm{~nm} ; p<0.001)$ (Fig. $2 B)$. Although this increased periodicity could contribute to the relative hypermyelination observed in such peripheral fibers, it is not sufficient to account for it. In the CNS, the thinner myelin observed in NFHLacZ transgenic mice was not associated with any alteration in interperiod spacing (11.1 $\pm 0.8 \mathrm{~nm})$ (Fig. $2 B)$.

Previously, a role for axonal neuregulin-1 type III (Nrg1 type III) in the control of PNS myelin thickness was reported. A potential role for Nrg1 type I in the control of CNS myelin thickness change in the number of Schwann cells associated with a unit length of fiber. Thus, if internodal lengths decrease in NFdeficient axons, the peripheral nerves in NFH-LacZ mice would maintain a larger-than-normal Schwann cell population. To estimate the size of the Schwann cell population, cross sections from mid L4 roots obtained from 2-week- and 3-month-old control and transgenic mice were examined by electron microscopy $(20,000 \times)$ and the number of myelinated axons and Schwann cell nuclear profiles were counted. In 2-week-old mice, Schwann cell nuclei were associated with 5.43 and $4.73 \%$ of the ventral root axons in control and transgenic samples, respectively. Equivalent 
Table 1. Quantification of myelin proteins by Western blot experiments

\begin{tabular}{lccc}
\hline & $\mathrm{Tg} / \mathrm{Ct}(\%)$ & \\
\cline { 2 - 4 } & Brain & Spinal cord & Sciatic nerve \\
\hline P0 & & $77 \pm 8^{*}$ \\
PLP & $105 \pm 2$ & $87 \pm 13$ & \\
MBP & $93 \pm 6$ & $111 \pm 15$ & $105 \pm 24$ \\
CNPase & $93 \pm 42$ & $94 \pm 19$ & $94 \pm 6$ \\
CD9 & $114 \pm 19$ & $101 \pm 25$ & $118 \pm 16$ \\
\hline
\end{tabular}

Quantification of myelin proteins by Western blot experiments. For each Western blot, values were normalized using tubulin signal, which is not modified in NFH-LacZ mice. The signal intensity of control samples was defined as $100 \%$. For each Western blot experiment, control to transgenic ratios were calculated. Ratios from at least three experiments were averaged and expressed as mean \pm SD. Tg, Transgenic; $\mathrm{Ct}$, control. * Values are significantly different from corresponding values of control mice $(p<0.05)$.

results were observed for control and transgenic dorsal roots, but, as a higher proportion of small caliber fibers support the sensory system, Schwann cell nuclear profiles were associated with a higher proportion of axons $(6.8 \%$ in control and $6.7 \%$ in transgenic samples). Because internodal length increases with maturation (Court et al., 2004), Schwann cell nuclei were encountered less frequently in the samples from 3-month-old mice. A ventral control root contained 954 fibers and 27 Schwann cell nuclear profiles $(2.83 \%)$ whereas the corresponding transgenic root contained 1037 fibers and 31 nuclear profiles (2.99\%).

To evaluate internodal distances directly, we also measured internodal lengths on teased sciatic nerve fibers. A typical teased fiber preparation is shown in Figure $3 A$. Although the distance between nodes in the two fibers is similar, the transgenic fiber has an obviously reduced caliber. As predicted, a linear relation between the diameter of myelinated fibers and internodal length in both control and transgenic sciatic nerves was observed (Fig. 3B). A linear regression of fiber diameter versus internodal length was calculated, which was significantly different from zero $(p<$ 0.001 ), with an $r^{2}$ value of 0.4356 and 0.3834 for control and transgenic samples, respectively. Note that for similar diameters, fibers from transgenic mice have internodes longer than those from control mice. When expressed as the ratio of internodal length versus fiber diameter, control samples had the predicted ratio approximating 100 , whereas in the transgenic sample this ratio was approximately doubled. Moreover, the internodal lengths measured for the sciatic nerves of 3-month-old mice revealed statistically similar mean lengths for transgenic and control fibers $(637 \pm 157 \mu \mathrm{m}$ vs $561 \pm 136 \mu \mathrm{m}$, respectively). Note also that the distribution observed in the transgenic sample indicates a tendency toward longer internodal distances in the reduced caliber fibers (Fig. 3C). These combined data demonstrate that internodal spacing is not reduced in PNS fibers maintaining normal amounts of myelin on caliber reduced axons.

To establish internodal lengths in the CNS, we measured internodal distances using serial cross sections. Because individual oligodendrocytes typically myelinate multiple axons and support a variable number of internodes, estimates of internodal distances based on the number of oligodendrocytes per length of a given CNS track would be error prone. Also, teased fibers are difficult to prepare reliably from CNS tracts obviating direct visualization of internodes. To overcome such issues, thoracic spinal cord segments from 3-month-old mice were embedded in Epon and 1000 serial $1-\mu$ m-thick sections were mounted in order and stained with toluidine blue. One hundred large caliber axons were followed through all sections. Nodes of Ranvier were recognized by a local reduction in axon caliber and disappearance of the myelin sheath (Fig. 3D). Internodal length was established according to the number of sections separating two nodes. The mean internodal length observed in control and transgenic samples was similar: $393 \pm 151 \mu \mathrm{m}(n=82)$ for control versus $387 \pm$ $154 \mu \mathrm{m}(n=86)$ for transgenic mice (Fig. $3 F)$. Moreover, a similar relationship between fiber diameter and internodal length was observed (Fig. $3 E$ ) with a positive linear regression, significantly different from zero $(p<0.001)$ with respective $r^{2}$ values of 0.3018 and 0.3531 . As observed in the PNS, for CNS fibers the ratio between internode length and axon diameter was $\sim 100$ in control and $\sim 200$ in transgenic samples. These data suggest that CNS fibers without NFs establish internodes of the same length that they would have achieved had their axonal diameters not been reduced by experimentally withholding the NF cytoskeleton. In addition, because the thickness of the myelin sheath is proportionately reduced in the CNS fibers of NFH-LacZ mice, these data also demonstrate that absolute myelin thickness is not of significance to the mechanism establishing nodal spacing.

\section{Node of Ranvier dimensions in fibers lacking axonal neurofilaments}

NF phosphorylation status correlates positively with interfilament spacing and increased axonal caliber in numerous normal and experimental circumstances. Notably, the reduced caliber of axons at nodes of Ranvier is associated with locally dephosphorylated NFs. Thus, it has been proposed that the NF phosphorylation status plays a role in controlling relative axonal dimensions in nodal and internodal segments (De Waegh et al., 1992). If this hypothesis is correct, axon calibers in both nodal and internodal domains in the NF-deficient NFH-LacZ fibers may be similar. However, large caliber reductions were observed at all of the nodal regions encountered in the spinal cord cross sections obtained from NFH-LacZ mice (Fig. 3D). Furthermore, we measured nodal calibers in subpopulations of fibers selected to have similar internodal calibers and again, the control and transgenic results were equivalent. The selected control sample of 172 fibers had a mean internodal caliber of $3.7 \pm 1.11 \mu \mathrm{m}$ and a mean nodal caliber of $1.07 \pm 0.35 \mu \mathrm{m}$ whereas the corresponding transgenic sample had a mean internodal caliber of $3.41 \pm 1.04 \mu \mathrm{m}$ and a mean nodal caliber of $1.06 \pm 0.32 \mu \mathrm{m}$ (Fig. $4 A$ ).

Nodal and internodal calibers also were estimated by measuring their dimensions on semithin transverse sciatic nerve sections (Fig. $4 A$ ). Sixty-one control fibers with an average internodal caliber of $3.93 \pm 1.06 \mu \mathrm{m}$ demonstrated an average nodal caliber of $0.59 \pm$ $0.14 \mu \mathrm{m}$. For the NFH-LacZ sample, the results were similar; 67 fibers with an average internodal caliber of $3.56 \pm 0.86 \mu \mathrm{m}$ had an average nodal caliber of $0.53 \pm 0.14 \mu \mathrm{m}$. Because axons with and without a local NF cytoskeleton reduce their calibers at nodes to the same extent, the mechanism establishing nodal dimensions appears to include axonal components other than NFs.

To determine whether a similar reduction in nodal axon caliber also occurs in NF-deficient PNS fibers, we evaluated longitudinal sections obtained from L4 ventral root. Figure 4, $B$ and $C$, shows an electron micrograph of a typical node of Ranvier encountered in an NF-deficient fiber. Despite the lack of NF throughout the axon profile, a marked decrease in caliber is observed at the node. Also, prominent electron dense transverse bands are observed between paranodal loops and the axolemma (Fig. $4 C$, arrowheads), suggesting that such nodes contain mature axoglial junctions.

Axonal calibers in small unmyelinated axons are influenced more by microtubules than NFs (Friede and Sarnorajski, 1970). As a denser microtubule array replaces the NF-deficient axoskeleton in myelinated axons of NFH-LacZ mice (Eyer and Peterson, 1994), microtubules might contribute disproportionately to axon calibers. If such compensation were unequal in internodes and at nodes, it 
might account for the relative decrease in nodal calibers. To investigate this possibility, we determined whether the microtubule density differed between internodal domains and the node of Ranvier (Fig. 4D,E). In the presence of axonal NF, the density of microtubules at the node of Ranvier in CNS fibers increases twofold when compared with the internode. Similarly, in NFdeficient axons, the density of microtubules at the node of Ranvier is increased when compared with the internode although to a lesser extent (1.3-fold). In PNS samples, microtubule density at the nodes of Ranvier was increased 2.8-fold in the presence of NF and 1.9-fold in the absence of NF.

Because NF deficient axons in the PNS recruit myelin sheaths that are inappropriately thick for their absolute calibers, novel organization of the myelin paranodal loops is required. Packaging supernumerary paranodal loops could be accommodated by extending the paranodes longitudinally, thus providing greater surface area for myelin lamellae to contact the axon or a subset of paranodal loops could terminate superficially failing to contact the axon surface. To distinguish between these possibilities, longitudinal sections through nodes in the L4 ventral roots were examined by electron microscopy. The longitudinal extent of the paranodal region was increased in transgenic fibers, although further ultrastructural abnormalities, including superficially terminating paranodal loops, were not observed.

We next used anti-Caspr labeling to establish the paranode/node/paranode (PNP) length/axon caliber ratios (Fig. $4 F$ ). Consistent with the ultrastructural findings, we observed a significantly increased ratio in NFH-LacZ sciatic nerves compared with controls (8.12 \pm 3.23 vs $4.35 \pm 1.56$, respectively; $p<0.001$ ). In contrast, the thinner CNS fibers in the NFHLacZ spinal cord with proportionally reduced myelin thickness demonstrated significantly reduced PNP length/axon caliber ratios $(6.06 \pm 2.00$ vs $7.64 \pm 2.89$ in NFH-LacZ and controls, respectively; $p<0.01$ ).

Immunolabeling for sodium channels in samples fixed with $4 \%$ PFA provided an alternate strategy to evaluate nodal dimensions. As defined by intense sodium channel labeling, nodal lengths were similar in both NFH-LacZ and control samples for both sciatic nerve $(1.2 \pm 0.5$ $\mu \mathrm{m}$ vs $1.2 \pm 0.4 \mu \mathrm{m})$ and spinal cord $(1.0 \pm 0.4 \mu \mathrm{m}$ vs $1.0 \pm 0.6 \mu \mathrm{m})$ samples. As a consequence of the reduced axon calibers in NFH-LacZ mice, the surface area of the nodal axolemma is reduced significantly in both the PNS ( $5.5 \pm 2.8 \mu \mathrm{m}^{2}$ vs $\left.8.7 \pm 4.3 \mu \mathrm{m}^{2} ; p<0.001\right)$ and CNS $\left(2.9 \pm 2.0 \mu \mathrm{m}^{2}\right.$ vs $\left.3.6 \pm 1.7 \mu \mathrm{m}^{2} ; p<0.01\right)$.

The molecular architecture of nodes of Ranvier is unaffected by the lack of axonal neurofilaments

Neuronal proteins localized at nodes of Ranvier are variously anchored to the axoskeleton. Sodium channels are linked to ankyrin $G$ (Lemaillet et al., 2003) that is bound to $\beta I V$-spectrin (Komada and

D
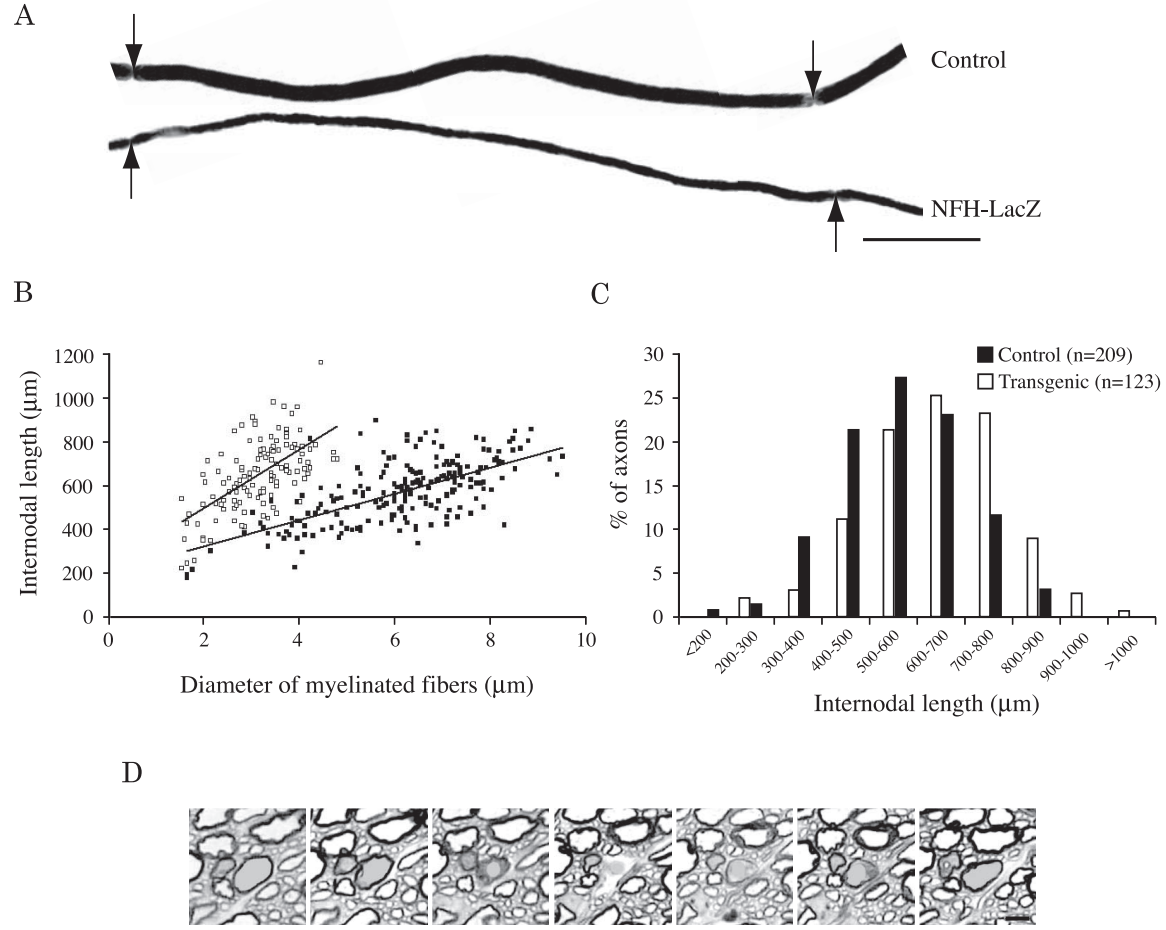

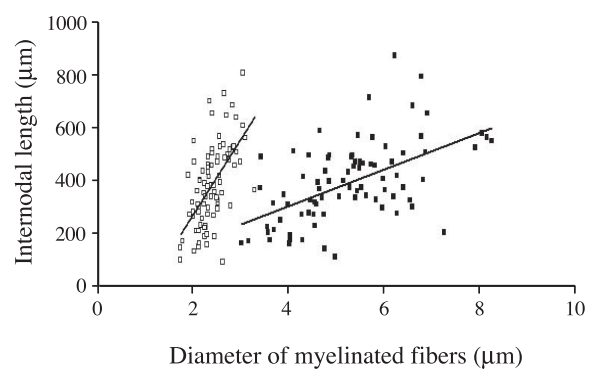

Figure 3. Despite the reduced axonal caliber, the internodal length is not affected in either the CNS and PNS from NFH-LacZ mice. $\boldsymbol{A}$, Teased myelinated fibers of sciaticnerve from control (top) and NFH-LacZ (bottom) mice. Arrows, Nodes of Ranvier. Note that although the diameter of the transgenic fiber is reduced, the internodal length is similar to the normal fiber. $\boldsymbol{B}$, Fiber diameter and internodal length measured in sciatic nerve of control (black squares) and NFH-LacZ (white squares) mice show a linear relationship between fiber diameter and internodal length. C, Internodal lengths are similar between control (black bars) and transgenic (white bars) sciatic nerves (561 \pm 136 m v $637 \pm 157 \mu \mathrm{m}$ ), despite a reduced axonal size in transgenic samples. D, Serial sections of spinal cord from control mice showing an axon (in blue) at the level of a node of Ranvier. E, Fiber diameter and internodal length measured in spinal cord of control (black squares) and NFH-LaCZ (white squares) mice. Both types of fibers show a linear relationship between fiber diameter and internodal length. $\boldsymbol{F}$ Internodal lengths are similar between control (black bars) and transgenic (white bars) spinal cords ( $393 \pm 151 \mu \mathrm{m}$ vs $387 \pm 154 \mu \mathrm{m}$ ) despite the reduced size of axons in transgenic samples. Scale bars: $A, 100 \mu \mathrm{m} ; \boldsymbol{D}, 2 \mu \mathrm{m}$.
Soriano, 2002). Similar interactions with the actin cytoskeleton occur at paranodal and juxtaparanodal domains, mediated through protein 4.1B (Denisenko-Nehrbass et al., 2003). Moreover, actin microfilaments are connected to intermediate filaments via linker proteins such as bullous pemphigoid antigen 1 neuronal and plectin (Foisner et al., 1988; Yang et al., 1996). Notably, mice expressing mutated $\beta \mathrm{IV}$-spectrin demonstrate NF abnormalities (Yang et al., 2004), suggesting that these molecules are functionally linked. To determine whether such interacting molecules accumulate normally in NFdeficient fibers, we used Western blot analysis.

First, to determine whether the absence of its NF binding partner could affect $\beta$ IV-spectrin accumulation, we evaluated the amount of $\beta I V$-spectrin present in sciatic nerves and spinal cords of 
4-month-old normal and transgenic NFH-LacZ mice. No difference was observed in either the bands detected or their relative intensities (Fig. 5A). In contrast, Western blot analysis showed that the levels of sodium channels, detected using a pan-Nav antibody, are increased in transgenic spinal cords and sciatic nerves (Fig. 5C). For spinal cord samples, similar results were observed with an antibody specific to Nav1.2, although the amount of Nav1.6 appeared to be normal. Relative accumulation of these isoforms was not determined in sciatic nerves because they accumulate to levels too low for accurate evaluation.

To determine whether the absence of axonal NF could affect the distribution of axolemmal proteins normally located at nodes, we used immunocytochemistry. Longitudinal sections from both PNS and CNS fibers were analyzed for sodium channels (pan-Na, Nav1.2, and Nav1.6), $\beta$ IV-spectrin, Caspr, and potassium channels (Kv1.1). Localization and immunocytochemical staining intensities appeared to be normal in the transgenic samples (Fig. 5B). Moreover, the normal switch in sodiumchannel isotype occurs as Nav1.2 is expressed in postnatal day 10 spinal cords (Fig. $5 B u, B v$ ), whereas in mature animals it is replaced by Nav1.6 (Fig. $5 B a, B b, B m, B n$ ). Finally, the localization of $\beta I V$-spectrin, Caspr, and Kv1.1 in sciatic nerves and spinal cords was similar between control and transgenic samples (Fig. 5B). Thus, NFs are not required for the appropriate localization or maintenance of these nodal proteins.

\section{The electrophysiological properties of neurofilament deficient axons are severely affected}

The altered morphology of axons in NFH-LacZ transgenic mice predicts major differences in their conduction properties. In the absence of changes in axoplasmic composition, the attenuated caliber of CNS and PNS axons and the maintenance of the internodal length would predict an increase in axonal resistance and, therefore, a longer latency to depolarization threshold at adjacent nodes. This should result in the slowing of conduction velocity, and to investigate this hypothesis, several stimulation/recording paradigms were applied to transgenic and control littermates. Conduction was evaluated at several sites of the PNS of adult mice (3- to 7-month-old). In one experiment, stimulus electrodes were placed on the distal tail with platinum recording electrodes placed between 40 and $50 \mathrm{~mm}$ more proximal on nerves exposed at the tail base. Both motor and sensory fibers were stimulated in this preparation, but the majority of the rapid response detected in this experiment is assumed to arise from large caliber myelinated sensory fibers rather than from antidromic conduction in stimulated motor fibers. Slower conduction velocities were observed in the caudal and tibial nerves of transgenic mice. At 3 months of age, transgenic mice conducted at only $65 \%$ the rate of normal mice $(24.7 \pm 1.8 \mathrm{vs} 38.2 \pm 0.9 \mathrm{~m} / \mathrm{s})$ (Fig. 6A). The amplitude of the compound action potential (CAP) in transgenic nerves also was attenuated (Fig. 6B).

Additional in vivo measurements were designed to evaluate the conduction properties of monosynaptic and polysynaptic pathways. In these experiments, we determined the latency and amplitude of motor evoked potentials. The large-caliber motor fibers in ventral spinal roots were brought to threshold using subcutaneous stimulating electrodes placed over the lumbar spinal cord. Recordings were obtained from electrodes inserted in the gastrocnemius muscle and its tendon. In both control and transgenic mice, biphasic activity of large amplitude was recorded. No significant differences were observed in the interval between initial and peak responses (1.21 vs $1.25 \mathrm{~ms}$ ) or in the amplitude of the evoked response, but relative conduction rates, revealed as the latency from stimulation to response, was slowed in transgenic mice (P. Lonchampt, unpublished observation).

Two additional stimulating and recording experiments were designed to investigate pathways with multiple synapses. The peripheral and central components of an auditory evoked response (Fig. 6C) and the primarily cortical response to direct electrical stimulation of the spinal cord at the thoracic level were evaluated (Fig. 6D). A succession of four distinct peaks, typical of auditory evoked potentials, were observed in control mice. Although $\mathrm{NFH}$-LacZ transgenic mice revealed a similar pattern, the peaks were less distinct and the latencies of the recognizable waves were prolonged (Fig. 6C). Somesthesic evoked potentials recorded in control mice present as a triphasic wave, corresponding to the activity of afferents to the spinal cord, followed by a more prolonged negative wave. In transgenic mice, the somesthesic evoked potentials were less defined, more variable, and had increased latencies. (Fig. 6D).

\section{Discussion}

NFs are essential for axons to achieve normal calibers (Cleveland et al., 1991). In the NFH-LacZ mice studied here, average axonal diameters in both the CNS and PNS are $\sim 50 \%$ of normal. However, the absence of axonal NF does not result in a population of fibers with identical calibers. Moreover, the extent of axon caliber reduction is not uniform across all axon populations, because smaller caliber axons show a more limited reduction (Jean and Fressinaud, 2003). These results show that although NFs play a fundamental role in axon radial growth, they do not act alone to determine the final caliber of axons.

An intact myelin sheath is necessary for NFs to be fully phosphorylated. Thus, any myelin disruption leads to local axonal NF dephosphorylation and a corresponding reduction in axonal caliber (DeWaegh et al., 1992; Kirkpatrick et al., 2001). Consistent with this relationship, NFs are poorly phosphorylated at the node of Ranvier and the axonal caliber is reduced (Mata et al., 1992). However, the relative extent of caliber reduction at nodes is similar between axons with and without NFs, indicating that molecules different from NFs participate in sculpting nodal dimensions.

Without axonal NFs, numerous compensatory mechanisms could contribute to axon radial growth (e.g., the increased density of the axonal microtubule (MT) cytoskeleton may affect axon calibers in the transgenic samples). If such increased MT accumulation is limited to internodal domains, it could account for the relatively reduced axon calibers seen at nodes. The density of MT observed in cross sections obtained from both nodal and internodal regions was significantly increased, strengthening the notion that factors other than NF accumulation are engaged in establishing the relative calibers of nodal and internodal domains in normal fibers. However, the extent of the MT density increase at nodes was not as large as that seen in control samples, leaving open the possibility that MT or other compensatory mechanisms might be involved. Alternatively, these size relationships could depend on physical properties of the axon; high amounts of F-actin, myosin, and mitochondria in paranodal loops could constrict nodal axons in an energy-dependent manner (Zimmermann, 1996, Landon and Hall, 1976). Because axons of increasing sizes are surrounded by proportionally increased number of myelin lamellae, the number of paranodal loops, and consequently the capacity to exert such constrictive pressure, could increase with increasing axon calibers.

Despite the axonal NF deficiency in NFH-LacZ mice, nodal, paranodal, and juxtaparanodal components are correctly localized. Furthermore, the developmental switch between Nav1.2 
A
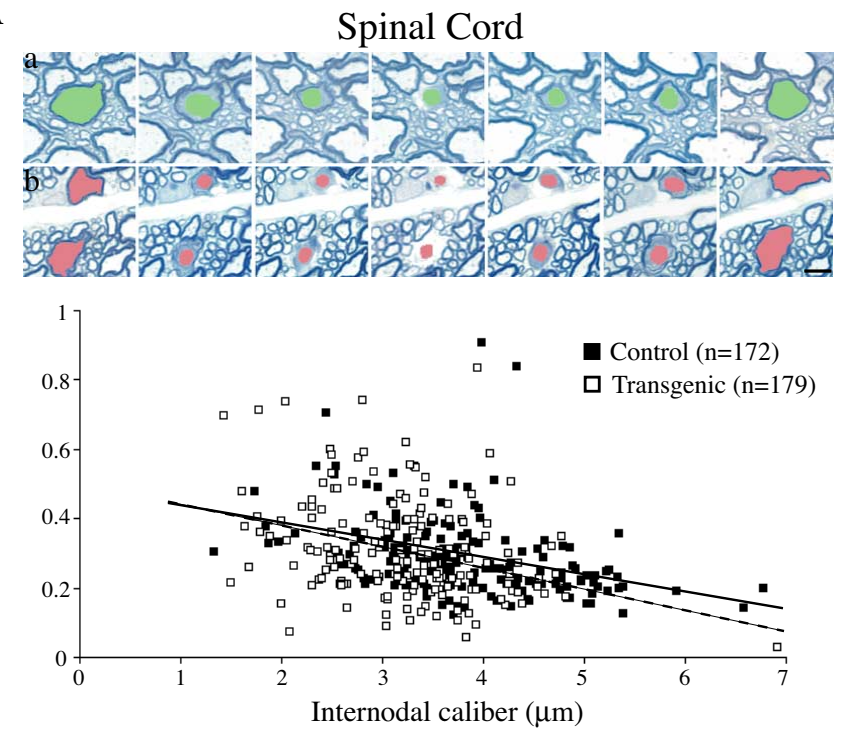

Sciatic Nerve
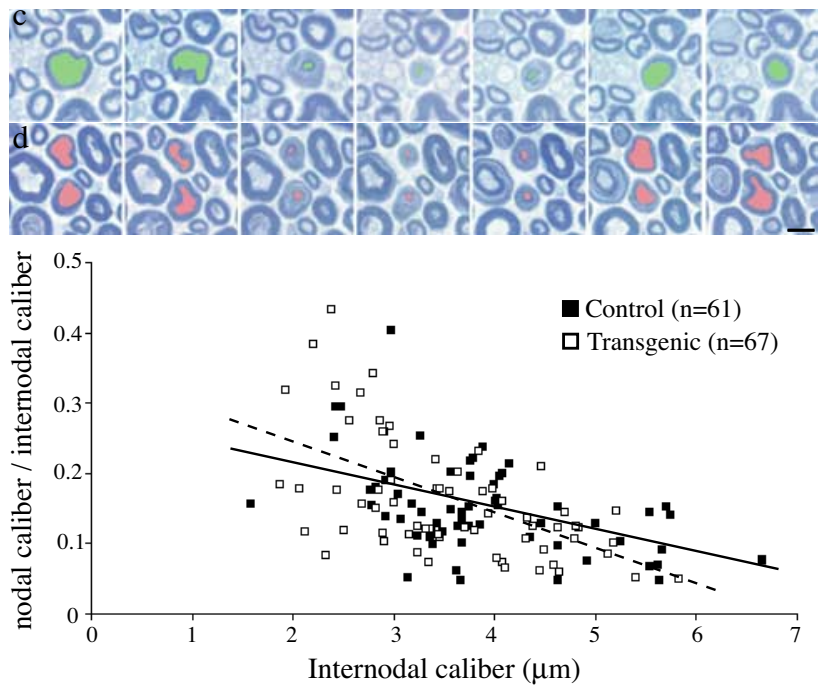

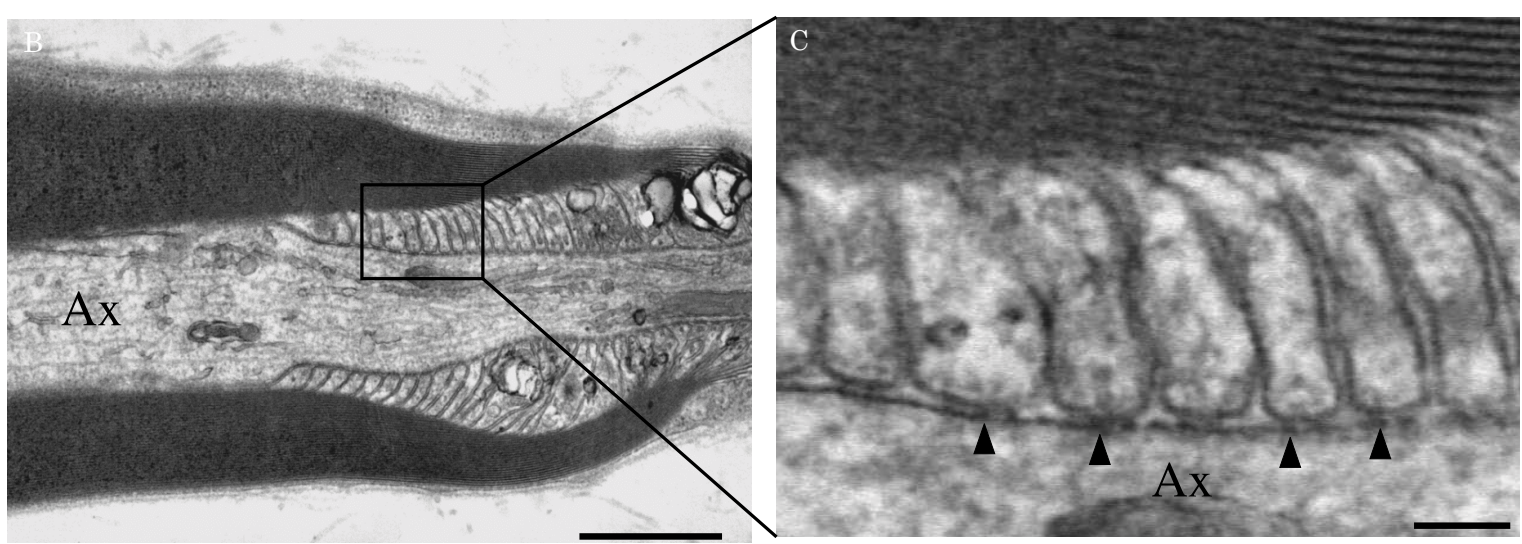

$\mathrm{D}$

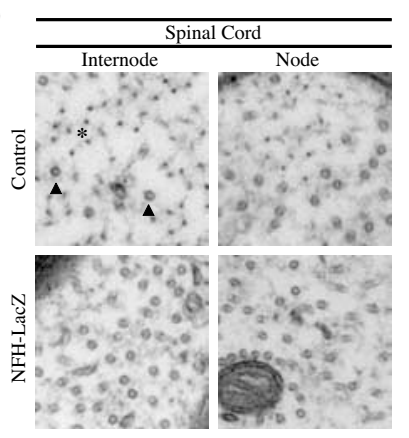

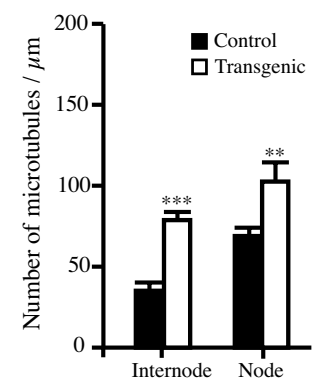

$\mathrm{E}$
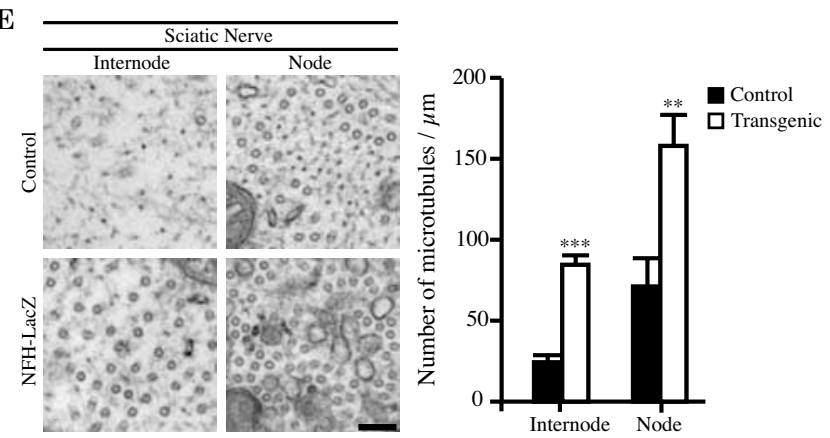

F

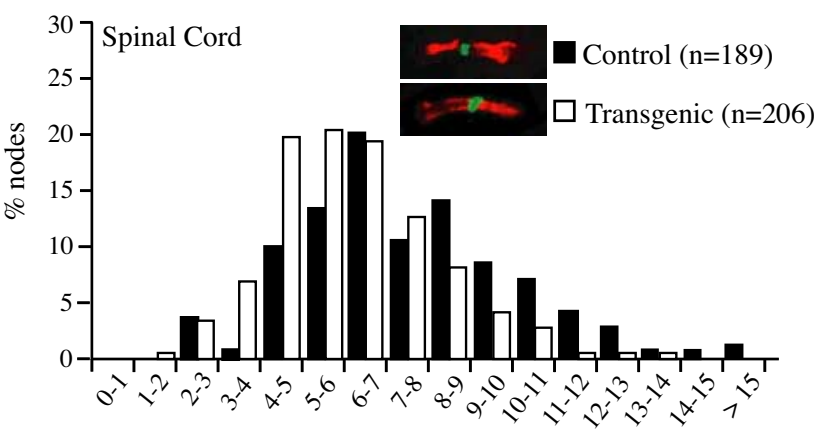

PNP length / axon caliber

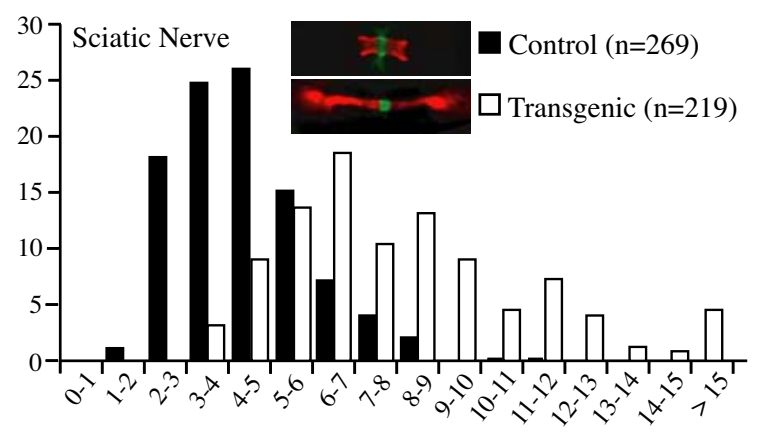

PNP length / axon caliber 
and Nav1.6 occurs on the appropriate schedule. These observations indicate that NFs are not required to position or anchor nodal components. At least for the process of nodal positioning, our observations are consistent with the suggestion that molecules present in myelinating cells may play a significant role in this process (Boiko et al., 2001; Eshed et al., 2005).

It was shown previously that Nrg1erbB signaling in PNS fibers plays an important role in controlling myelin thickness. Specifically, the amount of myelin formed is proportional to axonal Nrg1 type III levels (Michailov et al., 2004; Taveggia et al., 2005). Because normal amounts of PNS myelin are produced by NFH-LacZ mice, we expected Nrgl type III to be expressed to normal levels in PNS fibers. Whereas normal levels of the ErbB2 coreceptor were observed, Nrg1 type III accumulated only $50 \%$ of normal levels. Although as yet unresolved, a number of mechanisms could account for this unanticipated result; NF might be required to stabilize axonal Nrg1 type III or the reduced caliber of axons might limit docking space, again affecting stability. Another possibility could be that upstream control at transcriptional or translational levels might be influenced by signals emanating from the caliber-reduced axons. Alternatively, because Nrg1 type III activity is dispensable for maintenance myelin sheaths in the adult (Atanasoski et al., 2006), sufficient signaling to establish normal myelin levels might be achieved during early formation of myelin in these mice. Finally, Chen et al. (2006) showed that P0 gene expression is specifically regulated by $\mathrm{Nrg} 1$ type III. Thus, the reduction of axonal Nrg1 type III in the NFH-LacZ sciatic nerve may account for the observed reduction in $\mathrm{P} 0$ accumulation.

Axon electrical activity affects the supply of astrocyte-derived leukemia inhibitory factor, which in turn controls oligodendrocyte myelin elaboration (Ishibashi et al., 2006). Although illuminating this pathway exposes at least part of the mechanism through which the timing of myeli-
A

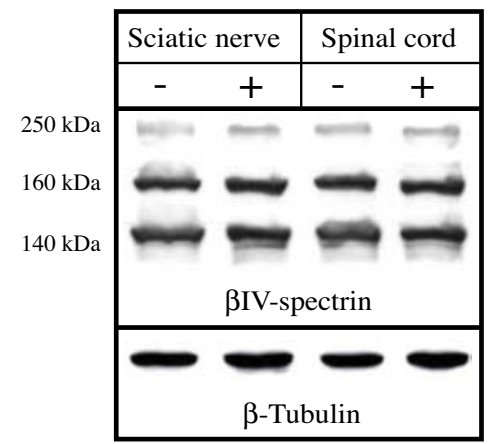

B
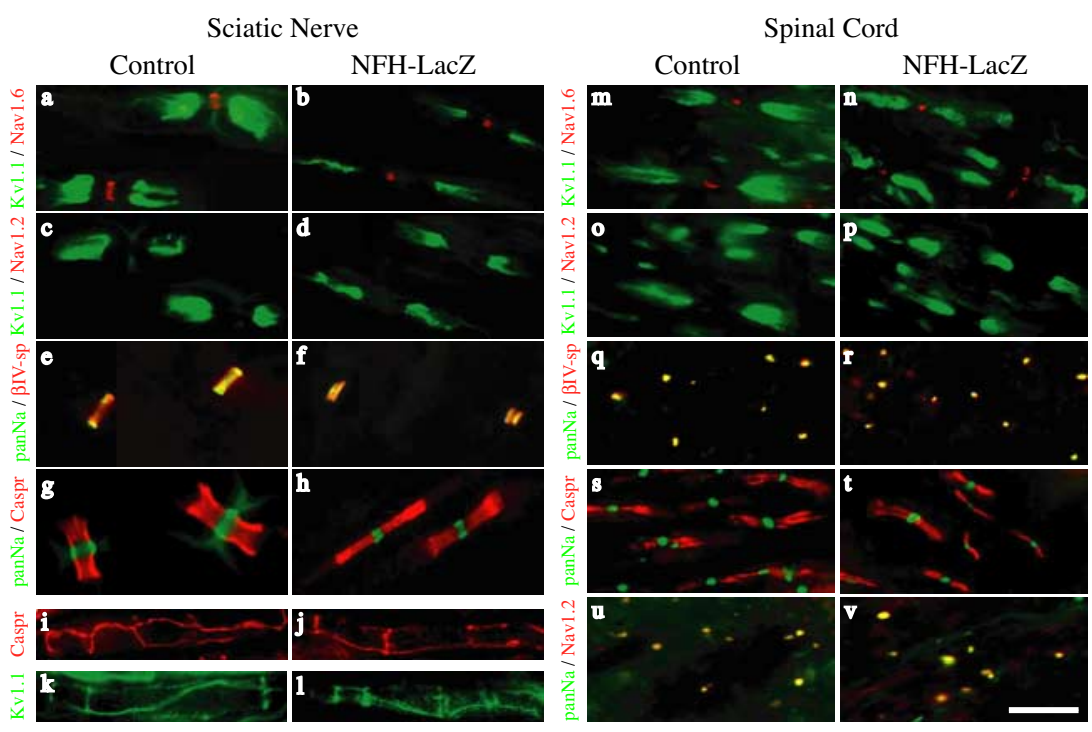

C

\begin{tabular}{|c|c|c|c|c|c|}
\hline \multicolumn{2}{|c|}{$\mathrm{Pan}-\mathrm{Na}_{\mathrm{V}}$} & $\mathrm{Na}_{\mathrm{v}} 1.2$ & $\mathrm{Na}_{\mathrm{v}} 1.6$ & \multicolumn{2}{|c|}{ Tubulin } \\
\hline+ & + & + & + & + & + \\
\hline- & $=\mathrm{CP}$ & 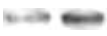 & $=1$ & 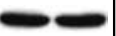 & \\
\hline Sp. Cord & Sciatic N. & Sp. Cord & Sp. Cord & Sp. Cord & Sciatic N \\
\hline
\end{tabular}

Figure 5. Molecular organization of nodes of Ranvier. $A$, Westerm blot analysis of $\beta \mathrm{IV}$-spectrin in 4-month-old control (-) and NFH-LaCZ(+) mice. Anti- $\beta \mathrm{IV}$-spectrin antibody recognized three bands of 140, 160, and $250 \mathrm{kDa}$. The lightest band corresponds to $\beta \mathrm{IV} \Sigma 6$-spectrin and the heaviest to $\beta \mathrm{IV} \Sigma 1$-spectrin. The amount of $\beta \mathrm{IV}$-spectrin is unchanged in transgenic sciatic nerve and spinal cord. Tubulin is used as an internal control. $\boldsymbol{B}$,Sciaticnerves $(\boldsymbol{a}-\boldsymbol{I})$ and spinal cord sections $(\boldsymbol{m}-\boldsymbol{v})$ from 4 -month-old $(\boldsymbol{a}-\boldsymbol{t})$ and postnatal day $10(\boldsymbol{u}, \boldsymbol{v})$ wild-typeortransgenicmicewere immunostained using antibodies to Caspr, pan-Nav channels, Nav1.2, Nav1.6, Kv1.1, and $\beta I V-$ spectrin. Remarkably, all of these components were correctly localized in both normal and transgenic fibers. However, in transgenic mice, the paranodes are longer in the PNS when compared with control samples. The internodal localization of Caspr and Kv1.1 is also normal in transgenic sciatic nerve $(i-I)$. The switch of sodium channels is correctly achieved in the absence of NFs because in spinal cord, Nav1.2 is present in nodes of postnatal day $10 \mathrm{NFH-LacZ}$ mice $(\boldsymbol{v})$, and then replaced by Nav1.6 in adult mice $(\boldsymbol{t})$. Scale bar, $10 \mu \mathrm{m}$. $\boldsymbol{C}$, The amount of voltage-gated sodium channels (pan-Nav) is increased in both the CNS and PNS of NFH-LacZ mice. The Nav1.2 amount is increased in transgenic spinal cord whereas the Nav1.6 amount is normal. Tubulin is used as an internal control.

Figure 4. Despite the absence of NFs in internodal segments, the axonal caliber is reduced at nodes of Ranvier. $\boldsymbol{A}$, Serial sections of spinal cord $(\boldsymbol{a}, \boldsymbol{b})$ and sciatic nerve $(\boldsymbol{c}, \boldsymbol{d})$ from 3 -month-old control $(\boldsymbol{a}, \boldsymbol{c})$ and NFH-LacZ mice $(\boldsymbol{b}, \boldsymbol{d})$. Despite the absence of axonal NF in internodal segments of transgenic samples, the extent of the axonal caliber reduction at the nodes of Ranvier is similar between transgenic and control fibers. Bottom, The level of axonal caliber reduction at nodes is more important for large fibers than for small fibers, both in control (black squares) and NFH-LacZ (white squares) samples, and in the PNS as well as in CNS. Regression lines are represented (full, control; dotted, NFH-LaCZ). B, C, Electron micrographs through the paranodal region (longitudinal section) from a 4-month-old NFH-LacZ mouse L4 ventral root. Axon (Ax) caliber is reduced despite the absence of detectable NFs. Note the presence of typical transverse bands (arrowheads) indicating the correct formation of the junctional complex normally formed between the axolemma and paranodal loops. $D, E$, Axons with NFs or deprived of NFs have an increased density of microtubules in both nodal and internodal domains, as observed on ultrathin sections of 3-month-old control and NFH-LacZ spinal cords (D) and sciatic nerves (E). Arrowheads point toward the MT whereas the asterisk indicates NF. Error bars indicate SEM. ${ }^{* *} p<0.01 ;{ }^{* * *} p<0.0001$. F, Ratio between PNP Iength and axon caliber was evaluated in sciatic nerve and spinal cord from control and NFH-LacZ mice using anti-Caspr labeling. This ratio is increased in transgenic sciatic nerves compared with control samples, suggesting that the length of paranodes is inappropriately long for these smaller axons. However, the ratio is slightly reduced in transgenic spinal cord. Scale bars: $A, 2 \mu \mathrm{m} ; \boldsymbol{B}, 1 \mu \mathrm{m} ; \boldsymbol{C}, 0.1 \mu \mathrm{m}$; (in $\boldsymbol{E}) \boldsymbol{D}, \boldsymbol{E}, 100 \mathrm{~nm}$. 
A

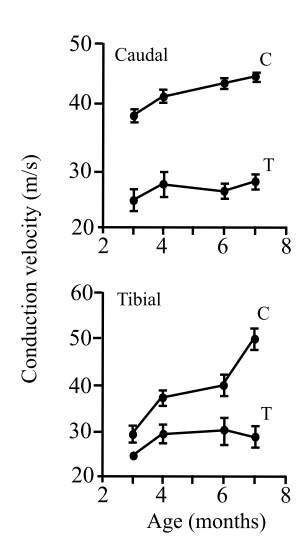

B

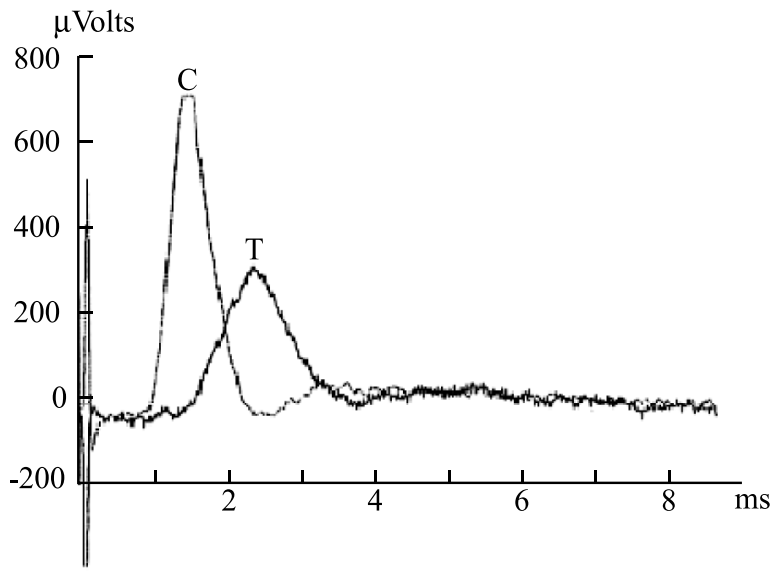

C
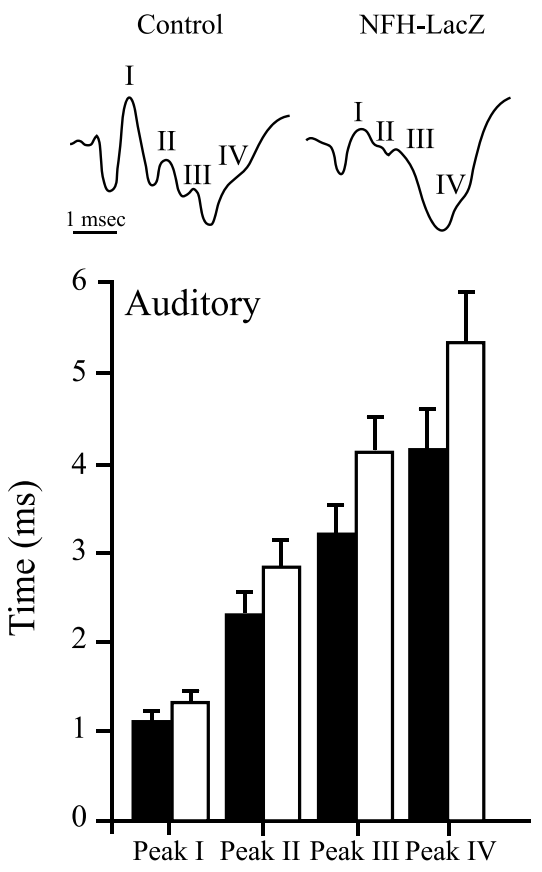

$\mathrm{D}$

Control
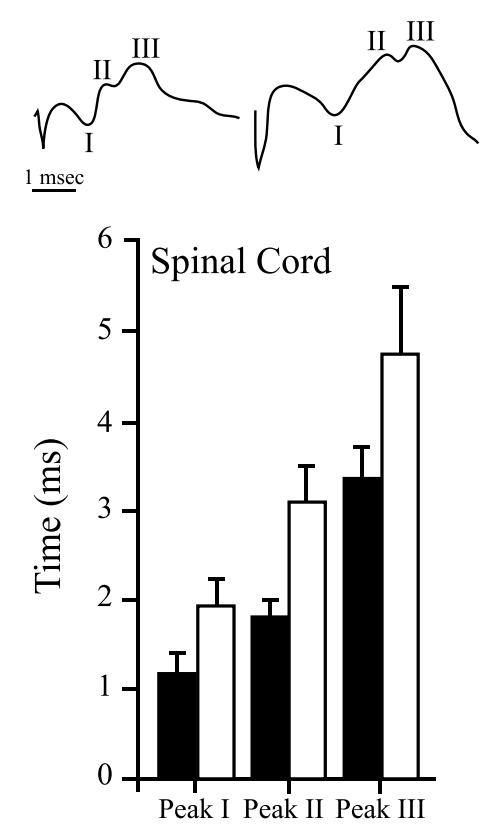

Figure 6. Electrophysiological analysis of NFH-LacZ mice. $\boldsymbol{A}$, Slower conduction velocities were observed in the caudal and tibial nerves of transgenic mice. Whereas the conduction velocity increases with age in normal animals, it does not increase similarly in transgenic mice. Error bars indicate SD. B, CAPs recorded from control (C) and transgenic (T) nerves. Note the decreased conduction velocity and amplitude in NFH-LacZ nerves. $\boldsymbol{C}, \boldsymbol{D}$, Latencies to the peaks were longer in transgenic mice when the auditive $(\boldsymbol{C})$ and somesthesic evoked potentials $(\boldsymbol{D})$ were analyzed. Error bars indicate SD. The top graphs represent typical recordings of evoked potentials in control and NFH-LacZ mice.

nation is controlled, the molecular mechanisms regulating the amount of myelin elaborated remain poorly defined. Our demonstration that Schwann cells and oligodendrocytes elaborate different amounts of myelin in response to a common axonal anomaly demonstrates that, whatever these control mechanisms are, they are not engaged in a similar manner by CNS and PNS fibers.

Internodal length correlates positively with axonal caliber (Fried et al., 1982) and this relationship is maintained in NFHLacZ mice. However, the absolute reduction in axon calibers of their CNS and PNS fibers is not associated with shortening of internodal lengths. Although the mechanism used by fibers to position nodes of Ranvier remains elusive, this result alone demonstrates that the absence of axonal NFs and alterations in the radial dimensions of axons and myelin sheaths are not disruptive.

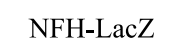

That NFs do not participate in nodal positioning is consistent with several observations. During development, axonal sodium channels aggregate at the ends of oligodendrocyte sheaths (Rasband et al., 1999; Boiko et al., 2001). In retinal ganglion cell axons, sodium channels are limited to nodes in the distal myelinated segment whereas in the unmyelinated proximal segment, their distribution is diffuse (Boiko et al., 2001). Also, deficiencies in laminin, dystroglycan, or gliomedin, which are localized to Schwann cell microvilli, affect the clustering of sodium channels (Saito et al., 2003; Eshed et al., 2005; Occhi et al., 2005). Moreover, internodal length in adults depends on the initial positioning of nodes of Ranvier and on the subsequent capacity of myelinated cells to elongate during growth (Court et al. 2004). If axons were to dictate nodal positioning, the fibers properties of NFHLacZ mice require that they must do so through a mechanism operating independently of axonal NFs, absolute axon calibers, myelin thickness, and normal conduction properties. Alternatively, if myelinating cells alone determine the localization of nodes of Ranvier, their elongation capacity must be controlled by axonal properties unrelated to NFs and absolute axon calibers. Consistent with this model and as seen in the fibers of NFH-LacZ mice, recent investigations have suggested that the thickness and length of the myelin sheath are controlled independently (Michailov et al., 2004).

The long-term stability of neuronal populations in NFH-LacZ mice provides an ideal model to investigate the electrophysiological consequences of absent axonal NFs (Eyer and Peterson, 1994). Optimum conduction velocities are achieved when internodal lengths are $\sim 100$ times the axonal caliber (Rushton, 1951; Friede and Beuche, 1985). In NFH-LacZ mice, internodal lengths are $\sim 200$ times the axon caliber and, according to Rushton (1951), such an abnormal ratio would increase internal resistance thus reducing capacity to activate sodium channels. This alone should lead to decreased conduction velocity and amplitude. Consistent with this model, lower conduction velocities are observed in several animal models characterized by reduced axon calibers (Sakaguchi et al., 1993; Zochodne et al., 2004). However, despite the normal axon calibers realized in $\mathrm{NFH}-/-$ mice, conduction velocity is markedly decreased (Kriz et al., 2000). Moreover, g ratios are markedly different in the CNS and PNS of NFH-LacZ mice, but similar alterations of conduction properties are observed. Together, these data suggest that axonal NF regulate conduction properties through mechanisms affecting fiber properties other than axon caliber or myelin thickness. One possibility is that medium-size neurofilament subunit and NFH, both heavily phosphorylated on 
their C-terminal tails, modulate ionic conductance, thus affecting the properties of axonal channels.

Nav1.2 is normally distributed along unmyelinated fibers. The increased accumulation of Nav1.2 in the CNS of NFH-LacZ mice could reflect higher per-fiber accumulation or increased numbers of unmyelinated fibers. Because the amount of myelin extracted from NFH-LacZ spinal cord is proportionally lower than the average myelin thickness, an increased number of unmyelinated CNS axons might exist in NFH-LacZ mice. However, normal numbers of myelinated axons were observed in the CNS and PNS of NFH-LacZ animals, requiring that any increase in unmyelinated fibers was not at the expense of the myelinated fiber population (Eyer et al. 1998). Alternatively, an increased density of Nav1.2 channels may be restricted to unmyelinated axons. Notably, Chen et al. (2006) observed that disruption of Nrg1erbB signaling leads to hypersensitivity and suggested that altered accumulation, localization, or function of sodium channels could be responsible. Thus, the $32 \%$ higher levels of erbB2 observed in NFH-LacZ spinal cord might be sufficient to elicit increased Nav1.2 accumulation.

This investigation shows that withholding NFs from the axonal compartment has dramatic, but different, secondary consequences on the size and composition of myelin sheaths in the PNS and CNS. Moreover, conduction properties are strongly affected despite apparently normal placement, organization, and physical dimensions of nodes of Ranvier. These unique fiber properties provide additional insight into the axon-glial relationships that modulate the development of mammalian fibers.

\section{References}

Arroyo EJ, Xu T, Grinspan J, Lambert S, Levinson SR, Brophy PJ, Peles E, Scherer SS (2002) Genetic dysmyelination alters the molecular architecture of the nodal region. J Neurosci 22:1726-1737.

Atanasoski S, Scherer SS, Sirkowski E, Leone D, Garratt AN, Birchmeier C, Suter U (2006) ErbB2 signaling in Schwann cells is mostly dispensable for maintenance of myelinated peripheral nerves and proliferation of adult Schwann cells after injury. J Neurosci 26:2124-2131.

Bhat MA, Rios JC, Lu Y, Garcia-Fresco GP, Ching W, St Martin M, Li J, Einheber S, Chesler M, Rosenbluth J, Salzer JL, Bellen HJ (2001) Axonglia interactions and the domain organization of myelinated axons requires neurexin IV/Caspr/Paranodin. Neuron 30:369-383.

Boiko T, Rasband MN, Levinson SR, Caldwell JH, Mandel G, Trimmer JS, Matthews G (2001) Compact myelin dictates the differential targeting of two sodium channel isoforms in the same axon. Neuron 30:91-104.

Boyle ME, Berglund EO, Murai KK, Weber L, Peles E, Ranscht B (2001) Contactin orchestrates assembly of the septate-like junctions at the paranode in myelinated peripheral nerve. Neuron 30:385-397.

Chen S, Velardez MO, Warot X, Yu ZX, Miller SJ, Cros D, Corfas G (2006) Neuregulin 1-erbB signaling is necessary for normal myelination and sensory function. J Neurosci 26:3079-3086.

Cleveland DW, Monteiro MJ, Wong PC, Gill SR, Gearhart JD, Hoffman PN (1991) Involvement of neurofilaments in the radial growth of axons. J Cell Sci [Suppl] 15:85-95.

Court FA, Sherman DL, Pratt T, Garry EM, Ribchester RR, Cottrell DF, Fleetwood-Walker SM, Brophy PJ (2004) Restricted growth of Schwann cells lacking Cajal bands slows conduction in myelinated nerves. Nature 431:191-195.

Denisenko-Nehrbass N, Oguievetskaia K, Goutebroze L, Galvez T, Yamakawa H, Ohara O, Carnaud M, Girault JA (2003) Protein 4.1B associates with both Caspr/paranodin and Caspr2 at paranodes and juxtaparanodes of myelinated fibres. Eur J Neurosci 17:411-416.

De Waegh SM, Lee VM, Brady ST (1992) Local modulation of neurofilament phosphorylation, axonal caliber, and slow axonal transport by myelinating Schwann cells. Cell 68:451-463.

Elder GA, Friedrich Jr VL, Margita A, Lazzarini RA (1999) Age-related atrophy of motor axons in mice deficient in the mid-sized neurofilament subunit. J Cell Biol 146:181-192.

Eshed Y, Feinberg K, Poliak S, Sabanay H, Sarig-Nadir O, Spiegel I, Berming- ham Jr JR, Peles E (2005) Gliomedin mediates Schwann cell-axon interaction and the molecular assembly of the nodes of Ranvier. Neuron 47:215-229.

Eyer J, Peterson A (1994) Neurofilament-deficient axons and perikaryal aggregates in viable transgenic mice expressing a neurofilament-betagalactosidase fusion protein. Neuron 12:389-405.

Eyer J, Cleveland DW, Wong PC, Peterson AC (1998) Pathogenesis of two axonopathies does not require axonal neurofilaments. Nature 391:584-587.

Foisner R, Leichtfried FE, Herrmann H, Small JV, Lawson D, Wiche G (1988) Cytoskeleton-associated plectin: in situ localisation, in vitro reconstitution, and binding to immobilized intermediate filament proteins. J Cell Biol 106:723-733.

Fried K, Hildebrand C, Erdelyi G (1982) Myelin sheath thickness and internodal length of nerve fibres in the developing feline inferior alveolar nerve. J Neurol Sci 54:47-57.

Friede RL, Beuche W (1985) A new approach toward analyzing peripheral nerve fiber populations. I. Variance in sheath thickness corresponds to different geometric proportions of the internodes. J Neuropathol Exp Neurol 44:60-72.

Friede RL, Samorajski T (1970) Axon caliber related to neurofilaments and microtubules in sciatic nerve fibers of rats and mice. Anat Rec 167:379-387.

Garcia ML, Lobsiger CS, Shah SB, Deerinck TJ, Crum J, Young D, Ward CM, Crawford TO, Gotow T, Uchiyama Y, Ellisman MH, Calcutt NA, Cleveland DW (2003) NF-M is an essential target for the myelin-directed "outside-in" signaling cascade that mediates radial axonal growth. J Cell Biol 163:1011-1020.

Giese KP, Martini R, Lemke G, Soriano P, Schachner M (1992) Mouse P0 gene disruption leads to hypomyelination, abnormal expression of recognition molecules, and degeneration of myelin and axons. Cell 71:565-576.

Hildebrand C (1972) Evidence for a correlation between myelin period and number of myelin lamellae in fibres of the feline spinal cord white matter. J Neurocytol 1:223-232.

Ishibashi T, Dakin KA, Stevens B, Lee PR, Kozlov SV, Stewart CL, Fields RD (2006) Astrocytes promote myelination in response to electrical impulses. Neuron 49:823-832.

Jacomy H, Zhu Q, Couillard-Despres S, Beaulieu JM, Julien JP (1999) Disruption of type IV intermediate filament network in mice lacking the neurofilament medium and heavy subunits. J Neurochem 73:972-984.

Jean I, Fressinaud C (2003) Spontaneous central nervous system remyelination is not altered in NFH-LacZ transgenic mice after chemical demyelination. J Neurosci Res 73:54-60.

Kaplan MR, Cho MH, Ullian EM, Isom LL, Levinson SR, Barres BA (2001) Differential control of clustering of the sodium channels $\mathrm{Na}(\mathrm{v}) 1.2$ and $\mathrm{Na}(\mathrm{v}) 1.6$ at developing CNS nodes of Ranvier. Neuron 30:105-119.

Kirkpatrick LL, Witt AS, Payne HR, Shine HD, Brady ST (2001) Changes in microtubule stability and density in myelin-deficient shiverer mouse CNS axons. J Neurosci 21:2288-2297.

Komada M, Soriano P (2002) $\beta$ IV-spectrin regulates sodium channel clustering through ankyrin-G at axon initial segments and nodes of Ranvier. J Cell Biol 156:337-348.

Kriz J, Zhu Q, Julien JP, Padjen AL (2000) Electrophysiological properties of axons in mice lacking neurofilament subunit genes: disparity between conduction velocity and axon diameter in absence of NF-H. Brain Res 885:32-44.

Laemmli UK (1970) Cleavage of structural proteins during the assembly of the head of bacteriophage T4. Nature 227:680-685.

Landon DN, Hall S (1976) The myelinated nerve fibre. In. The peripheral nerve, pp 1-105. New York: Wiley.

Lemaillet G, Walker B, Lambert S (2003) Identification of a conserved ankyrin-binding motif in the family of sodium channel alpha subunits. J Biol Chem 278:27333-27339.

Mata M, Kupina N, Fink DJ (1992) Phosphorylation-dependent neurofilament epitopes are reduced at the node of Ranvier. J Neurocytol 21:199-210.

Michailov GV, Sereda MW, Brinkmann BG, Fischer TM, Haug B, Birchmeier C, Role L, Lai C, Schwab MH, Nave KA (2004) Axonal neuregulin-1 regulates myelin sheath thickness. Science 304:700-703.

Occhi S, Zambroni D, Del Carro U, Amadio S, Sirkowski EE, Scherer SS, Campbell KP, Moore SA, Chen ZL, Strickland S, Di Muzio A, Uncini A, Wrabetz L, Feltri ML (2005) Both laminin and Schwann cell dystrogly- 
can are necessary for proper clustering of sodium channels at nodes of Ranvier. J Neurosci 25:9418-9427.

Poliak S, Peles E (2003) The local differentiation of myelinated axons at nodes of Ranvier. Nat Rev Neurosci 4:968-980.

Rao MV, Campbell J, Yuan A, Kumar A, Gotow T, Uchiyama Y, Nixon RA (2003) The neurofilament middle molecular mass subunit carboxylterminal tail domains is essential for the radial growth and cytoskeletal architecture of axons but not for regulating neurofilament transport rate. J Cell Biol 163:1021-1031.

Rasband MN, Peles E, Trimmer JS, Levinson SR, Lux SE, Shrager P (1999) Dependence of nodal sodium channel clustering on paranodal axoglial contact in the developing CNS. J Neurosci 19:7516-7528.

Rushton WA (1951) A theory of the effects of fibre size in medullated nerve. J Physiol (Lond) 115:101-122.

Rydmark M (1981) Nodal axon diameter correlates linearly with internodal axon diameter in spinal roots of the cat. Neurosci Lett 24:247-250.

Saito F, Moore SA, Barresi R, Henry MD, Messing A, Ross-Barta SE, Cohn RD, Williamson RA, Sluka KA, Sherman DL, Brophy PJ, Schmelzer JD, Low PA, Wrabetz L, Feltri ML, Campbell KP (2003) Unique role of dystroglycan in peripheral nerve myelination, nodal structure, and sodium channel stabilization. Neuron 38:747-758.

Sakaguchi T, Okada M, Kitamura T, Kawasaki K (1993) Reduced diameter and conduction velocity of myelinated fibers in the sciatic nerve of a neurofilament-deficient mutant quail. Neurosci Lett 153:65-68.
Sherman DL, Brophy PJ (2005) Mechanisms of axon ensheathment and myelin growth. Nat Rev Neurosci 6:683-690.

Stampfli R (1954) Saltatory conduction in nerve. Physiol Rev 34:101-112.

Taveggia C, Zanazzi G, Petrylak A, Yano H, Rosenbluth J, Einheber S, Xu X, Esper RM, Loeb JA, Shrager P, Chao MV, Falls DL, Role L, Salzer JL (2005) Neuregulin-1 type III determines the ensheathment fate of axons. Neuron 47:681-694.

Waxman SG (1975) Electron-microscopic observations on preterminal fibers in the oculomotor nucleus of the cat. With special reference to the relation between axon diameter and myelin thickness in mammalian gray matter. J Neurol Sci 26:395-400.

Yang Y, Dowling J, Yu QC, Kouklis P, Cleveland DW, Fuchs E (1996) An essential cytoskeletal linker protein connecting actin microfilaments to intermediate filaments. Cell 86:655-665.

Yang Y, Lacas-Gervais S, Morest DK, Solimena M, Rasband MN (2004) $\beta$ IV spectrins are essential for membrane stability and the molecular organization of nodes of Ranvier. J Neurosci 24:9230-9240.

Yin X, Baek RC, Kirschner DA, Peterson A, Fujii Y, Nave KA, Macklin WB, Trapp BD (2006) Evolution of a neuroprotective function of central nervous system myelin. J Cell Biol 172:469-478.

Zimmermann H (1996) Accumulation of synaptic vesicle proteins and cytoskeletal specializations at the peripheral node of Ranvier. Microsc Res Tech 34:462-473.

Zochodne DW, Sun HS, Cheng C, Eyer J (2004) Accelerated diabetic neuropathy in axons without neurofilaments. Brain 127:2193-2200. 